\title{
Carnets
}

Revue électronique d'études françaises de l'APEF

Deuxième série - $20 \mid 2020$

Imaginaire(s) du Voyage

\section{Les imaginaire(s) du voyage à travers un projet de recherche création en danse}

\section{Biliana Vassileva}

\section{Q OpenEdition}

1 Journals

\section{Édition électronique}

URL : http://journals.openedition.org/carnets/12286

DOI : 10.4000/carnets.12286

ISSN : 1646-7698

Éditeur

APEF

\section{Référence électronique}

Biliana Vassileva, "Les imaginaire(s) du voyage à travers un projet de recherche création en danse », Carnets [En ligne], Deuxième série - 20 | 2020, mis en ligne le 30 novembre 2020, consulté le 20 avril 2021. URL : http://journals.openedition.org/carnets/12286; DOI : https://doi.org/10.4000/carnets. 12286

Ce document a été généré automatiquement le 20 avril 2021.

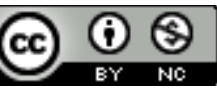

Carnets est mis à disposition selon les termes de la licence Creative Commons - Atribution - Pas d'utilisation commerciale 4.0 International. 


\title{
Les imaginaire(s) du voyage à travers un projet de recherche création en danse
}

\author{
Biliana Vassileva
}

\section{Introduction: « Drifting/a la dérive » de l'ouest vers l'est...}

1 Cette étude est centrée sur les imaginaires du voyage en danse comme art chorégraphique. Elle aborde plus précisément la posture académique des chercheurs et des chercheuses impliqués dans des projets de recherche création. Ceci implique à la fois la maîtrise de plusieurs savoirs théoriques, et les activités assidues d'immersion empirique en danse, en chorégraphie, en performance... Les exemples donnés par l'auteure de voyages, transformés en projet de recherche-création, sont eux-mêmes le résultat de quelques rencontres significatives, avec des chorégraphes et des danseurs, qui mélangent des sources d'inspiration occidentales et d'Asie. Il s'agit d'un " bricolage » de techniques de danse, de processus d'apprentissage, de transformations de répertoire local de danse.

2 L'étude va présenter ce processus de création, qui engage des savoirs interculturels en danse, dans le cadre d'un projet d'ancrage universitaire, Drifting/A la dérive, développé par l'auteure. Ce projet est construit sur le principe de métissage de références littéraires, d'arts visuels et d'exploration du mouvement de danse. Il permet d'analyser comment un voyage opère comme quête d'éclairage intuitif de soi, tel que le décrit Fernando Pessoa dans Le livre de l'intranquillité: "Mon âme est comme un orchestre caché ; je ne sais pas quels instruments résonnent et jouent en moi, cordes et harpes, timbales et tambours. Je ne peux me connaître que comme une symphonie. » (Pessoa, $1982: 76)$

3 Le projet Drifting/A la dérive est développé depuis 2012, à ce jour, et représente une série cohérente de courtes vidéos ${ }^{1}$. Chacune d'elles est faite au cours des voyages sur le 
principe d'imprégnation par des pratiques performatives locales. Ainsi, l'étude proposée questionne à la fois l'utilité de l'invention et l'établissement institutionnel d'une méthodologie de recherche-création. Ce type de méthodologie encourage des approches esthétiques de la recherche académique comme " practice as research » (pratique comme recherche). Le projet approfondit l'idée de l'imaginaire en danse et dans le champ performatif par le biais de mouvement improvisé. Il est souvent une réaction très spontanée aux nouveaux environnements traversés en Occident et en Asie. Il est soutenu au niveau théorique par le processus de lecture et de documentation, plus particulièrement le dossier « On Poetics and Performance » du journal Performance Research (Allsopp \& Kreider, $2015: 1-12$ ).

4 L'étude, accomplie par la production finale de courtes vidéos, collaborations avec une artiste visuelle - Victoria Donnet, et avec un créateur sonore - Guillaume Tiger, trace la transformation d'images, les transitions entre lumière et obscurité, la vibration de la main qui filme, le geste artisanal qui capte les trajets imprévisibles des sensations nomades. Chaque vidéo est composée par "dérives ", qui conduisent vers des rencontres interculturelles et vers des possibilités renouvelées à « se mettre en scène » par une caméra fugitive. Il s'agit d'un mélange les réalités alternatives, inspirées par le travail du danseur/ de la danseuse, en voyage...

5 «Le virtuel possède une pleine réalité, en tant que virtuel ", affirme Deleuze (1968: 269). Dans le cadre du projet Drifting, cette réalité est composée avec l'aide du montage audiovisuel et de musique expérimentale. Il s'agit de proposer un autre rapport au réel, loin d'un compte rendu avec prétention d'objectivation, ce qui serait l'équivalent d'un documentaire La fabrication d'une réalité alternative est plus proche à celle décrite par Que par des voies sensoriels: "Les réalite's 'virtuelles' ne sont pas irréelles, elles possèdent une certaine réalite notre rétine et les secousses que les simulateurs nous infligent» (Quéau, $1995: 15$ ). L'inventivité kinesthésique et la créativité onirique dans Drifting opèrent par le principe d'associations ce qui nous éloigne des structures singulières qui caractérisent le virtuel des sciences et des disciplines: "Si structures il y a dans l'œuvre d'art, ce sont des pluralités de structures, toujours co-construites entre l'auteur et le lecteur ou le spectateur, dans un processus de recherche qui ne ressemble en rien au vécu plein et originaire de la première lecture ou du premier spectacle. Deleuze décrit le virtuel comme un lambeau, tandis que les arts n'offrent comme virtualités que des fils d'Ariane au mieux enchevêtrés quand ils ne sont pas inextricables » (Noël, 2019). Dans ce sens, nous pouvons nous référer à Pierre Lévy et son concept de virtualisation. Plus qu'une dématérialisation, le numérique opère une "déterritorialisation » selon Lévy (1995), une perte de l'espace tel qu'on le connait. La virtualisation est alors un processus qui transforme et élargit nos possibilités réelles.

6 Le projet Drifting/A la dérive a été présenté par Its Liquid dans des expositions d'art contemporain en Italie (Bari), Espagne et Pologne, dans des festivals de performance SIME (Semaine Internationale de la musique électroacoustique) de l'Université de Lille, Pile ou Frasque co-organisé sur Le regard du cygne/Le générateur, à Paris, en mars 2019, dans des créations scéniques avec d'autres artistes contemporains, (Kate McDonald, Guillaume Tiger...), dans des colloques pour la valorisation du patrimoine culturel, organisé par Territorialités françaises, par le Paris College of Art sur le thème de Contemporary Images Collection, le 29 avril $2019^{2}$, et dans le cadre d'autres événements, 
publications et communications, de la plate-forme en recherche esthétique inter culturelle and other events, LangArts, Paris.

\section{Imaginaires d'artistes chorégraphiques aux croisements inter culturels}

7 L'usage de l'imagination ou des idées originales pour créer quelque chose de nouveau peut être défini comme une forme de créativité. Quelles sont les conditions propices pour encourager ce type de processus ? D'où vient la capacité de produire ou de s'en servir d'idées originales et inhabituelles ? Une grande partie de l'étude repose sur des rencontres régulières avec des styles traditionnels de danse d'Asie, transformés par des principes chorégraphiques occidentaux. Au cours de ces voyages et recherches dans plusieurs pays asiatiques (Chine, Bali, Taiwan, Thaillande, Malaisie, Corée de Sud, e.a.), l'auteure a rencontré des danseurs et des danseuses, ainsi que des chorégraphes, entraînés en formes anciennes codifiées de danse.

Mais en même temps, trop de règles peuvent anéantir la créativité, l'ingénuité et l'intuition de ces artistes à établir un dialogue entre ce qu'ils et ce qu'elles savent déjà, avec ce qui est rencontré sur le chemin, régi par le phénomène de globalisation et l'avènement des nouvelles technologies. Par exemple, la danseuse et la chorégraphe d'origine chinoise Kehua Li, ancienne membre de Beijing Dance/LTDX ${ }^{3}$, peut passer plusieurs heures sur le web, en improvisant d'une manière ludique pour dénicher de nouveaux gestes de chute au croisement de techniques d'arts martiaux locales et des explorations anti-gravitaires, provenant de la danse contemporaine occidentale ${ }^{4}$.

Une des caractéristiques de l'imaginaire en voyage est la capacité de percevoir le monde sous des lumières nouvelles et surprenantes, de trouver des configurations " cachées » dans le sens des métaphores interculturelles. Ceci permet de créer des liens entre des phénomènes disparates à première vue, et de générer des solutions. Pour aborder plus précisément l'art de l'imaginaire en danse, je peux évoquer le travail de la danseuse et chorégraphe balinaise Kadek Puspasari ${ }^{5}$ qui déconstruit des récits de dance théâtre d'Indonésie dans son solo Panji's Uchrony, par sa double formation en danse contemporaine occidentale et danse traditionnelle indonésienne. 


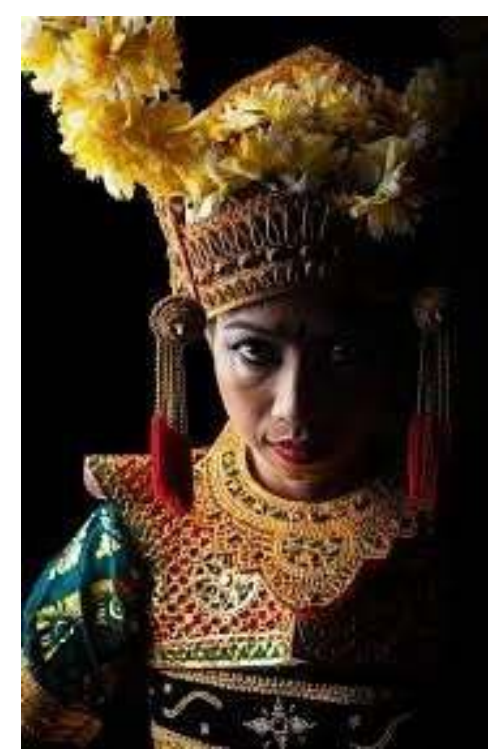

Kadek Puspasari, répertoire traditionnel, crédit : Iyan Xavier.

10 l'introduction d'éléments abstraits, et change des scénarios bien connus par sa plongée soudaine et prolongée dans des états sensoriels de corps et par ses interactions conviviales avec le public ${ }^{6}$.

11 La tradition de danse à Bali est, actuellement, entièrement développée sur la base de styles traditionnels, propres à l'île et transmis de génération à génération, sans grand changement. Les histoires qui les composent ressemblent à des contes de fées, peuplés de personnages magiques et aristocratiques. Il y a très peu, ou presque pas, de cours et de spectacles de danse contemporaine sur l'île, d'où le besoin de l'auteure en tante danseuse et chercheuse, qui suit des entraînements locaux en danse traditionnelle (étude de répertoires et incarnation de figures emblématiques), à trouver un certain état de " laisser aller " à la fois à l'intérieur, et à l'extérieur de formes codifiées apprises, par des pratiques autonomes d'improvisation ${ }^{7}$.

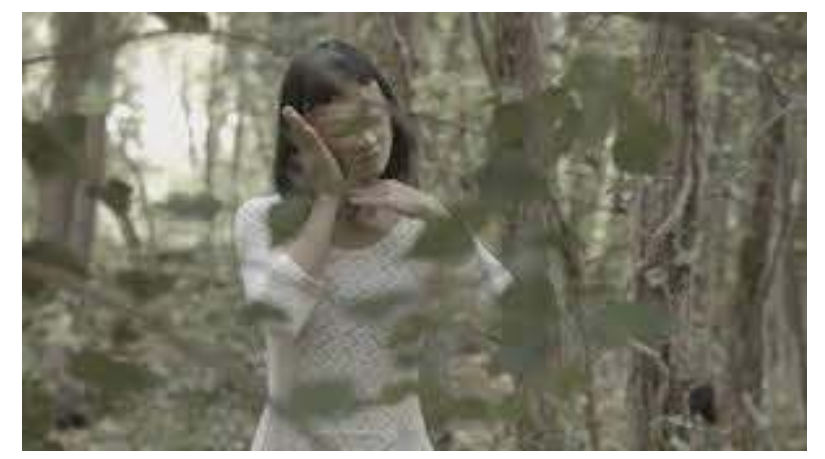

Kadek Puspasari, répertoire contemporain, crédit : Clément Thuriot
Cette tradition de danse est une sorte de colonne vertébrale culturelle de Bali. Sa ferme
maintenance et le faible intérêt pour des formes chorégraphiques contemporaines
répondent à la nécessité de l'île à préserver son identité. Entourée par des îles, régies
par la religion musulmane, Bali est la seule île hindouiste dans l'archipel d'Indonésie, ce
qui permet une culture du corps plus libre. 
13 D'une manière similaire, Thaïlande, un pays voisin, évolue par les marges possibles d'une tradition de danse, bien préservée, à des approches nouvelles, particulièrement sensibles et réactives, inspirées du contact avec des formes chorégraphiques occidentales importées. Le dialogue entre le passé et le présent dans la culture de la danse thaïlandaise est très actif. Il peut surprendre par des transitions inattendues entre des cours en danse traditionnelle qui se transforment vite et imperceptiblement en apprentissage de cabaret occidentalisé, populaire parmi les communautés artistiques de lady boys locaux et l'industrie de divertissement, qui fleurit dans les îles du Sud. Pour ce type d'imaginaire, qui évolue par des échanges interculturels entre l'Asie et l'occident, je pourrais me référer à la définition de Maria Popova, qui évoque une « force combinatoire »: " ... c'est notre capacité à puiser dans notre réservoir « interne » de ressources - savoir, intuition, information, inspiration et tous les autres éléments qui peuplent nos esprits - ce que nous avons accumulé pendant des années seulement en étant présents, vivants et éveillés au monde, - et à les combiner par des manières nouvelles extraordinaires. » (Popova, 2019:1)

Une autre définition de l'imaginaire proposée par Rollo May se réfère au long processus de création chorégraphique comme "quelque chose de nouveau en vie. La créativité demande de la passion et d'engagement. Elle ramène dans notre conscience ce qui a été, auparavant, caché et indique les nouveautés de la vie. Cette expérience de conscience amplifiée est une extase » (May, 1976:1). Cette " extase », en tant que « conscience amplifiée ", mène vers l'émergence dans ce qui est évoqué par, et invoqué dans, le langage du mouvement Gaga (Israel), sous l'instruction à " make your own creature " / inventer ta créature à toi ». (Vassileva, 2016:5)

15 Ce type de créature apparaît dans le travail d'une autre chorégraphe et danseuse d'Asie, Sun a Lee ${ }^{8}$, par le mélange d'éléments de danse et de yoga classique coréen et des procédés d'improvisation structurée de la danse contemporaine occidentale. Son solo Un-Cover (2019) est construit sur un autre principe d'imaginaire, décrit par Henry Rollins, qui consiste à " interpréter quelque chose qui est vue ou est un fruit d'expérience, et le transformer d'une manière différente de sa forme originelle " (Rollins, 2019 : 1). La traversée de la scène commence par une chaise, en position assise, méditative et frémissante, et finit par se dissoudre dans un noir absolu. D'abord, la danseuse fait un avec son masque mystérieux, venu des forêts lointains et animé par des cheveux longs et un corps mouvementé, entièrement couvert. Progressivement elle se défait du masque, comme si elle s'était affranchie d'une possession, qui semble perdurer malgré tout, et disparait de dos par une marche languissante...

Dans tous ces cas cités, le recours à des imaginaires du voyage en danse aide à ouvrir de nouvelles possibilités vers une abondance chorégraphique au lieu de se tenir à des limitations et à des lacunes. Il est étroitement lié avec un autre type d'imaginaire, plus pragmatique, pour les danseurs et les danseuses qui interprètent des propositions chorégraphiques d'inspiration interculturelle.

\section{Apprentissage de répertoire : des perspectives d'imaginaire du voyage de l'ouest et de l'est}

L'imaginaire dans le travail de certains chorégraphes, qui ont vécu au croisement de plusieurs environnements culturels, mène à l'invention de nouveaux langages : un 
brassage ( melting pot ») de cultures de danse, parfois divergentes. L'ontologie de ce processus nécessite une cartographie précise de leurs sources d'inspiration : quelles formes de danse de l'ouest et de l'est ont-ils et elles connu sur leur chemin ? Du point de vue des chercheurs et des chercheuses engagées dans des terrains immersifs, ceci permet, aussi, une analyse incorporée et détaillée, de " comment " ces sources s'hybrident, et comment les danseurs et les danseuses qui interprètent ce type de travail, peuvent glisser d'une mouvance vers une autre par des trajets multiculturels.

Cette partie de l'étude s'appuie sur une vision panoramique de quelques inventions de nouveaux langages hybrides de danse, qu'on peut qualifier d'interculturels, en s'appuyant, aussi, sur le discours des chorégraphes renommés comme Lin Hwai Min, fondateur de Cloud Gate Dance Theater of Taïwan, actuellement succédé par Cheng Tsung Lung ; Ohad Naharin, fondateur du langage du mouvement Gaga en Israël ; Xie Xin pour Xie Xin Dance Theater et Tao Ye pour Tao Dance Theater en Chine... Dans leur travail, apparaissent des processus d'improvisation, inspirée par des pratiques occidentales, qui évoluent sur les bases de traditions millénaires d'autres techniques du corps méditation, tai-chi... La méthodologie de recherche de l'auteure inclut étude bibliographique, terrains immersifs in situ, pratiques assidues d'incorporation et de recherche-création, et bien sur le voyage comme déplacement géographique réel (Vassileva \& Laureillard, 2016 : 419-429).

19 Pour illustrer l'analyse possible du travail d'imaginaire en danse, par des sources inter culturelles au cours de l'apprentissage, de l'interprétation, et par la suite dans la transcription de ce type de répertoire, voici quelques notes de terrain pour un solo de Mamootot (2003) d'Ohad Naharin, proposé dans un atelier d'étude de répertoire en 2019, ouvert au grand public, auquel l'auteure a participé en tant que danseuse :

\section{Notes de terrain par l'auteure, 2019}

La variation commence avec une remontée d'une posture repliée au sol vers une arabesque. Au lieu d'une simple projection spatiale vers le haut, cette transition peut inclure des roulements de muscles de l'abdomen... L'arabesque est une figure reconnaissable dans le vocabulaire du ballet occidental, mais ici la manière de le performer et faire fondre dans un plié profond qui engage une jambe enroulée sur l'autre est plus proche de l'esthétique des formes de danse, provenant d'Asie. L'arabesque en soi est très étirée, par ce qu'on appelle en Gaga « des forces horizontales » (" horizontales forces »), qui dégoulinent d'énergie douce, avec les bras tendus en avant dans un geste ambigu de donner et de recevoir à la fois... Il y a aussi un travail supplémentaire de la sensibilité de la peau...

Ensuite, en équilibre sur les deux jambes enroulées l'une sur l'autre, la colonne vertébrale commence à tourner lentement. Le « twist » étire peu à peu la peau du ventre et du sternum, tandis que le dos est nourri par des vagues qui se propagent vers le haut et qui permettent à garder l'axe vivant. Chaque posture ou transition doivent être repliées par un flux ("flow») pour faire en sorte que chaque mouvement déborde d'une manière presque inévitable dans le suivant. Dans ce type de continuum, qui est déjà travaillé et installé dans le corps du danseur et de la danseuse dans l'entraînement au préalable, tout commencement demeure doux, même les plus aigus.

Le mouvement suivant est un pas soudain, qui est aussi un étirement de la jambe qui travaille. Il y a plusieurs options à faire ce mouvement : de l'activation du bas du dos, nécessaire pour les "jetés » ou les «frappés » en ballet occidental au relâchement de la même partie du corps, pour une qualité plus fluide, qui dans les arts énergétiques d'Asie où il est aussi une préparation immédiate vers le mouvement suivant. C'est très important à souligner que dans le langage du 
mouvement Gaga il n'y a pas de divisions binaires de ce qui provient de l'ouest ou de l'est, mais il s'agit d'un amalgame perpétuel, qui dans l'interprétation de la proposition chorégraphique (la mienne incluse) est faite selon des choix individuels, en accord avec un savoir-faire préaccumulé.

Un adoucissement similaire de la cage thoracique est nécessaire pour le chargement abrupt suivant, avec le cœur qui s'érige vers le ciel. Sans cet état de détente dans le haut du corps, assez différente de la tonicité amplifiée, exigée dans le ballet et la danse moderne occidentale, la transition suivante sur demi-pointes sera presque impossible, ou avec un grand risque de déséquilibre.

Le mouvement suivant contient un des paradoxes spécifiques au Gaga qui est de tenir et de fondre simultanément. Il est suivi par un " tilt ", qui rappelle des figures de jazz, et une soudaine courbe du dos, qui rappelle des figures de danse moderne ; les deux peuvent hypothétiquement être une résonance lointaine de l'héritage d'Alwin Ailey, une des sources potentielles d'inspiration dans la genèse du langage chorégraphique...

La conception progressive de cette partition, au cours de l'apprentissage du solo par l'auteure, montre comment une pratique interculturelle en danse, accumulée par des expériences et des voyages, faits au préalable, peut nourrir le processus d'apprentissage et de recherche de la matière proposée. C'est important de souligner que le langage du mouvement Gaga, conçu comme un « melting pot » inter culturel en soi, permet ce type de jeu d'activation de ressources multiples.

21 Peaufiner des danses par des bribes d'incorporation et des clés à des codes locaux, collecter au cours de voyages, apporte un autre type d'imaginaire dans le champ académique de "practice as research» (" pratique comme recherche »). Cette capacité à connecter l'ensemble des savoir-faire, qui à première vue, n'ont rien en commun, peut devenir un besoin fondamental pour une chercheuse académique, qui régulièrement traverse les frontières interculturelles.

\section{La genèse et les principes d'évolution du projet Drifting/A la dérive}

Le projet de recherche création Drifting/A la dérive a commencé en 2012, avec un premier voyage à Bali, pour la création d'un court documentaire, soutenue par la plateforme académique LangArts, essentiellement dédiée aux travaux de recherche en esthétique interculturelle comparative Asie/Occident. Ce documentaire trace la collaboration de l'auteure avec la danseuse et pédagogue locale de répertoire traditionnel Debix Lupz Witha. Il est construit sur le principe de tranches explicatives ou poétiques, mais cette hybridité du projet, renouvelé par la suite, lui a rapidement donné une allure plus créative. 


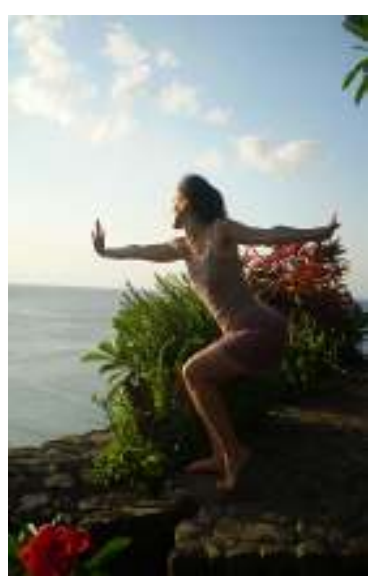

Biliana Vassileva (image et crédit), projet de recherche création « Drifting », Bali

l'élaboration d'une recherche structurée par approche empirique, sont étroitement liées avec sa longue pratique de danseuse, et le processus d'évolution de sa conscience kinésique, ainsi que ses compétences technicises. Le processus de création est articulé autour l'intention d'improviser, et plus tard de composer par le biais d'un montage visuel, une narrative de voyage qui est un ressenti kinesthésique, plutôt qu'une pensée rationnelle. Collecter des sensations de renaissance permet aux images, liées aux 
émotions plus sombres, à les transformer en mouvance et à les dévier de leurs origines. Les vidéos deviennent des carnets de voyages, de journaux de mémoire sensorielle. Cette mémoire, comparée à des constructions de signification, qui parfois les contractent, semble plus résistante dans le cadre de réalités culturelles alternatives... Parfois, la physicalité même de l'image va au-delà de l'intention initiale, et fait avancer l'histoire par sa propre poétique ; autrement dit le processus créatif possède des structures internes, dynamiques et génératrices de vitalité, plus proche du fonctionnement d'écriture de la poésie, que de celle de la prose... Ceci peut procurer une nouvelle raison et trajectoire pour le retour du voyage, par la déconstruction/ reconstruction du point ou l'urgence de départ.

Dans un voyage et sa transformation en vidéo danse, il n'y a pas de pression à tout capter, selon le principe de la flânerie de Baudelaire (1885) - se promener d'une manière nonchalante dans un environnement urbain ou autre, proche de l'état de " farniente ». Ce principe est préservé dans tous les volets du projet. Ce type de flânerie est la source de juxtapositions surprenantes d'image et d'imaginaires. Elle permet à osciller entre des temporalités différentes et à condenser des espaces divergents. L'événement qui est décrit, par la suite, obtient un statut ambigu d'être une fiction, composée par éléments réels. Ceci résonne avec la citation annoncée comme fondation théorique d'une possible perception du projet :

Il y a une poétique de l'édition. Maintenant nous vous invitons dans cette espace de poétique et de performance (votre entrée en matière, un autre commencement N.A.) pour suivre le double hélix qui fait ses méandres entre le conventionnel et le radical, le méthodologique et l'expérimental, le critique et le créatif. Et dans votre traversée (un pas, un saut, un vol...), nous vous invitons à trouver vos connexions et en faire votre interprétation. Il y a une poétique de lecture. (Allsopp \& Kreider, $2015: 3)$

\section{Perspectives anthropologiques, féministes et autres..., des imaginaire(s) du voyage en danse}

Dans le projet de recherche création Drifting/A la dérive le voyage devient un processus d'invention de nouvelle créativité d'agencement (" agency ») cinématographique, articulant, à la fois, les besoins professionnels de la chercheuse, et les besoins personnels de la danseuse/auteure. Cette invention nécessite, aussi, d'une nouvelle posture en recherche qualitative et post qualitative et d'un nouveau paradigme.

Par le regard sur les réflexions de la caméra en miroir comme matériel visuel à étudier, l'auteure a obtenu plus de conscience de " comment naviguer ", tout en se laissant aller "à la dérive ». L'observation de soi dans un processus de création féminin a longtemps été suspect d'une action potentiellement narcissique en opposition de la représentation de la femme domestiquée dans un espace socialement clos et réduit à l'intimité et à la modestie. Donc, même quand le sujet principal de la danse filmé est l'auteure, il y a une attention spéciale portée à l'environnement qui permet l'émergence du mouvement. Cette attention élargit la perspective et la possibilité de nouvelles découvertes et redéfinition de soi, par la promiscuité interculturelle...

Il est important ici de souligner la dimension relative de tout type d'éducation en danse ou somatique. Chaque retour de Thailand ou de Bali, ou d'un autre " ailleurs ", qui démarre le processus d'assemblage de la matière collectée et incorporée, en courts carnets de voyage, permet à l'auteure de se redéfinir selon le nouveau savoir inter 
culturel acquis en danse. Mais chaque nouveau départ contient aussi le risque d'un " dérapage ", puisque malgré tout le savoir et expertise, " être intelligent est relative selon les circonstances et ce qui nous entoure » (King, $2019: 156)$.

31 Ce type de savoir peut être acquis uniquement par des interactions locales, par « la conscience du regard des hôtes, et l'éducation réciproque obtenue " (King, 2019 : 140). Ceci nécessite une ouverture à l'altérité et une disponibilité spécifique, avant toute procédure analytique - « l'entraînement du cœur à voir l'humanité de l'autre » (King, 2019 : 102)... C'est pour cette raison que l'anthropologie culturelle aborde les « êtres humains dans le contexte de leur ethnos, les spécificités des sociétés et des communautés - nations, groupes ethniques, tribus, races, - par lesquelles il nous semble ils se débrouillent » (King, 2019: 115). L'esthétique interculturelle comparative, qui en dérive et qui est le fondement de ce projet en recherche création, aborde les œuvres d'art, émergente dans des contextes spécifiques de rencontres interculturelles. Ce savoir est en changement perpétuel, et voilà pourquoi je ne peux que poser des repères pour le processus créatif du projet, sans le fixer ou réduire à un certain nombre de thèmes...

L'expérience éphémère d'un voyage stimule à la fois des surfaces et des profondeurs, mais bien sûr en comparaison avec des séjours de longue durée, qui sont exigés dans le champ de l'anthropologie, la méthode créative de collecter des sensations comme prélèvement des « saillances » est plus spontanée et inclut un travail imaginaire. Elle est plus proche du champ de l'esthétique, qui ne prétend pas à révéler des "vérités ", surtout quand ceci engage un travail sensitif et sensible du corps en danse, et de ses usages. Malgré tout, une multiplicité de "vérités » profondément subjectives émerge par le jeu d'associations d'idées de nature visuelle, sonore et intellectuelle au cours du montage et de la création musicale.

Dans ce sens, le déplacement géographique est une condition obligatoire pour l'évolution du projet. Suite à la globalisation, plusieurs formes d'art de l'est sont " importées » et modifiées selon les besoins de l'ouest, et selon la vision occidentale de ce qu'une forme d'art ou un travail en danse doit être, comme évoqué plus haut. Voilà pourquoi partir "à la dérive » et à la rencontre des "vrais experts " devient indispensable : « les vrais experts - ceci veut dire ceux qui ont vraiment utilisé, dans un lieu et un temps bien spécifique ... » (King, 2019: 66). Le projet de recherche création est construit sur la base d'un bricolage à la fois artisanal et savant : « la combinaison de voyage, trouvailles d'artefacts, apprentissage de langues, et chasse aux os » (King, $2019: 66)$.

\section{BIBLIOGRAPHIE}

ALLSOPP Ric \& KREIDER Kristen (2015). « On Poetics and Performance ». Performance Research Volume » vol. 20. Oxfordshire : Routledge, pp. 1-12.

BAUDELAIRE, Charles (1885). Le Peintre de la vie moderne. Paris : Calmann Lévy. 
BOLENS, Guillemette (2008). Le Style des gestes : corporéité et kinésie dans le récit littéraire. Lausanne : BHMS.

DELEUZE, Gilles (1968). Différence et répétition. Paris : Presses universitaires de France.

GODARD, Hubert (2006). « Des trous noirs with Patricia Kuypers ». Nouvelles de danse, $n^{\circ} 53$, Bruxelles : Contredanse, pp. 56-75.

KING, Charles (2019). Gods of the Upper Air. New York : Doubledge.

LANGARTS (2013). Notions esthétiques : résonance entre les arts et les cultures. Paris : L'Harmattan. LEVY, Pierre (1995). Qu'est-ce que le virtuel ? Paris : La Découverte.

MASSIN, Marianne (2001). Les figures du ravissement. Paris: Grasset.

MAY, Rollo (1976). The Courage to Create. London : Collins.

NOËL, Dominique (2019). « Le virtuel selon Deleuze », conference pour Intellectica. Revue de

l'Association pour la Recherche Cognitive.

PESSOA, Fernando (1982 ; 2002). The Book of Disquiet, trans. Richard Zenith. UK: Penguin Classic.

POPOVA, Maria (2019). Brainpickings, Consulté le 21.12, 2019, URL www.brainpickings.org, date de d'accès 01/06/2020.

QUEAU, Philippe (1995). «Le virtuel : un état du réel ». Virtualité et réalité dans les sciences, Paris :

Éditions Frontière.

ROLLINS, Henry (2019). Brainpickings, Consulté le 21.12, 2019. URL: www.brainpickings.org.

VASSILEVA, Biliana (2016). « Dramaturgies of Gaga Bodies: Kinesthesia of Pleasure ». Danza e Ricerca, N 8 E-journal, Laboratorio di studi, scritturi, visioni. Italy : University of Bologne. URL : https:// danzaericerca.unibo.it/article/view/6605, date de d'accès 01/06/2020.

VASSILEVA, Biliana \& LAUREILLARD, Marie (2016). « L'écriture chorégraphique spiralée du Cloud Gate Dance Theater ». La scène mondiale aujourd'hui : des formes en mouvement, CIRRAS (Centre International de Réflexion et de Recherche sur les Arts du Spectacle), ouvrage collectif dir. F. Quillet, L'univers théâtral. Paris : L'Harmattan, pp. 419-429.

VASSILEVA, Biliana. Projet de recherche création Drifting/A la dérive. URL : https://

bilidanse.wixsite.com/website-2 ?fbclid =IwAR0DvP37p3MqSQBsVtmM_1wkvURnouVIv8DC7GCDYrW7A5b0wyi3W9IWhg, date de d'accès 01/06/2020.

\section{NOTES}

1. Site web: https://bilidanse.wixsite.com/website-2? fbclid=IwAR0DvP37p3MqSQBsVtmM_1wkvURn-ouVIv8DC7GCDYrW7A5b0wyi3W9IWhg

2. https://www.paris.edu/graduate-symposium-2019/

3. BeijingDance/LDTX est une compagnie de danse contemporaine, fondée par Willy Tsao dans Beijing en 2005. C'est la première compagnie professionnelle de danse avec statut indépendant du gouvernement.

4. https://www.youtube.com/watch ?v =5LRK2V--vQg

5. Kadek Puspasari a étudié dans l' Indonesian Institute of The Arts Surakarta, et actuellement gère sa propre compagnie de danse Pantcha Indra en France.

6. https://www.youtube.com/watch ?v =fNnQHc3KzcE 
7. https://www.youtube.com/watch ?v=qTqoQ2nDCXk - Bali Luwak, 2019, Projet de recherche création « A la dérive ".

8. Sun a Lee vit à Paris, et développe ses projets individuels SunadanSE. Elle danse aussi pour la Cie Le Guetteur - Luc Petton.

9. Site web: https://bilidanse.wixsite.com/website-2? fbclid=IwAR0DvP37p3MqSQBsVtmM_1wkvURn-ouVIv8DC7GCDYrW7A5b0wyi3W9IWhg )

\section{RÉSUMÉS}

Cette étude est centrée sur les imaginaires du voyage en danse comme art chorégraphique. Elle aborde plus précisément la posture académique des chercheurs et des chercheuses impliqués dans des projets de recherche création. Ceci implique à la fois la maîtrise de plusieurs savoirs théoriques, et les activités assidues d'immersion empirique en danse, en chorégraphie, en performance... Les exemples donnés par l'auteure de voyages, transformés en projet de recherche création, sont eux-mêmes le résultat de quelques rencontres significatives, avec des chorégraphes et des danseurs, qui mélangent des sources d'inspiration occidentales et d'Asie. Il s'agit d'un "bricolage " de techniques de danse, de processus d'apprentissage, de transformations de répertoire local de danse.

creativity, intercultural research creation, dance, performance, visual arts

The study approaches the concept of «travel imaginaries " " as choreographic and performance activity. It addresses the need of contemporary dance artists to travel, blending Eastern and Western dance techniques. The main focus is on an interplay of intercultural knowledge within the development of an academic based research creation project, called Drifting/A la dérive, developed by the author. It combines literature, visual arts and dance movement. The project takes place(s) from 2012 till today, and consists of a coherent series of videos, based on traveling and performance practice in East/West destinations. Its methodology of creative "travel imaginaries » constructions encourages « practice as research » approaches to aesthetics.

\section{INDEX}

Mots-clés : imaginaire, voyage, danse, recherche création

\section{AUTEUR}

\section{BILIANA VASSILEVA}

CEAC - Université de Lille

bilidanse[at]gmail.com 